\title{
La question agraire au Venezuela de 1493 aux années actuelles
}

The Agrarian Question in Venezuela, from 1493 to Current Years

\section{Olivier Delahaye}

\section{(2) OpenEdition}

\section{Journals}

\section{Édition électronique}

URL : http://journals.openedition.org/economierurale/2433

DOI : $10.4000 /$ economierurale. 2433

ISSN : 2105-2581

\section{Éditeur}

Société Française d'Économie Rurale (SFER)

\section{Édition imprimée}

Date de publication : 5 décembre 2009

Pagination : 115-128

ISSN : 0013-0559

\section{Référence électronique}

Olivier Delahaye, "La question agraire au Venezuela de 1493 aux années actuelles », Économie rurale

[En ligne], 313-314 | Septembre - décembre 2009, mis en ligne le 05 décembre 2011, consulté le 21 avril 2019. URL : http://journals.openedition.org/economierurale/2433 ; DOI : 10.4000/ economierurale.2433 


\section{La question agraire au Venezuela de 1493 aux années actuelles}

Olivier DELAHAYE • Facultad de Agronomía, Universidad Central de Venezuela. Maracay, Venezuela

\section{Introduction}

A près une relative éclipse lors des années 1990, la question agraire est revenue au premier plan des préoccupations des organismes multinationaux (Deininger, 2003). Sans qu'il puisse exister de corrélation plausible avec ce retour, elle a aussi repris une place importante au Venezuela, tant dans les analyses socioéconomiques que sur la scène politique, depuis l'élection du président Chávez en 1998 (De Long, 2005). Elle semblait pourtant avoir été maîtrisée, dans une large mesure, après les redistributions foncières massives effectuées dans le cadre de la loi de réforme agraire de 1960 et qui avaient marqué une brusque accélération du rythme historique de formation des droits de propriété. Parallèlement, on observait une baisse prononcée des demandes paysannes de terre présentées au long des quatre dernières décennies $\mathrm{du} \mathrm{XX}^{\mathrm{e}}$ siècle. Cependant les années 2000 voient une résurgence du débat et des luttes agraires, qui ne peut être exclusivement liée aux changements de la conjoncture politique nationale, même si ceux-ci jouent un rôle important. Cet article se propose de revisiter brièvement la question agraire au Venezuela, entendue comme la formation et l'évolution des droits de propriété (formels ou informels) sur les terrains agricoles. Suivant Soro et Colin (2004), cette étude utilise le concept de droits de propriété «dans une acception de sciences sociales et non juridiques, au sens d'actions autorisées, socialement légitimées, sur un bien ». Elle se place dans une perspective de long terme, avant d'observer l'évolution récente, depuis la fin de la dernière dictature en 1958 ; la continuité observée dans les résultats des mesures foncières prises depuis 2001 est relevée, qui peut sembler paradoxale au regard des déclarations de rupture des responsables gouvernementaux actuels vis-à-vis des administrations précédentes. Cette continuité peut partiellement s'expliquer par la prévalence historique des institutions informelles dans la régulation du foncier ; dans ce sens, le travail insistera sur l'importance de la formation et la transmission informelle des droits de propriété, fréquentes au Venezuela, comme dans beaucoup de pays d'Amérique latine (Deininger, op.cit.).

\section{Une rétrospective de la question agraire au Venezuela}

\section{Les déterminants historiques}

Le cadre qui détermine les structures foncières actuelles a été fixé par les bulles papales dictées par Alexandre VI en 1493 (Delahaye, 2001) ${ }^{1}$. Elles attribuèrent la propriété des «terres découvertes et à découvrir » aux couronnes d'Espagne et du Portugal, sans évoquer les droits des communautés précolombiennes, qui furent ainsi rejetés dans des limbes juridiques dont ils ne sortent que très tardivement et partiellement. Depuis cette date, la formation des droits fonciers privés à partir des terres publiques ${ }^{2}$ s'est effectuée au Venezuela, comme dans toute l'Amérique latine, selon deux voies :

1. Cette section historique s'appuie sur Delahaye (2001).

2. Les terres de la couronne sont devenues baldíos (terres de la nation) au moment de l'Indépendance. Celles-ci forment la majorité des terres publiques à l'heure actuelle. Les autres sont essentiellement les terrains d'origine privée affectés à la réforme agraire, et les ejidos (terres à la sortie du village) qui appartiennent aux municipalités. 
- Une voie légale, formelle, c'est-à-dire la délivrance de titres fonciers.

Sous la colonie, ce sont les mercedes, cédées en propriété privée par l'Administration coloniale aux quelques personnes qui avaient accès à cette dernière. Elles concernent un terrain déterminé (souvent sans précision) sur le titre correspondant. La couronne concédait aussi quelques resguardos (portions de terre) à certaines communautés indigènes. Inaliénables, ils étaient la propriété collective de celles-ci. Enfin la couronne donnait aux municipalités des terrains ejidos, eux aussi inaliénables, de propriété communale. Il n'existe pas de données vérifiables sur l'ensemble des dotations coloniales.

Après l'indépendance (1821), il s'agit tout d'abord des « avoirs militaires » répartis entre soldats et officiers des armées républicaines, dont l'importance n'est pas connue avec précision et qui ont fait l'objet de trafics qui les firent aboutir dans les mains des caudillos, les chefs militaires qui se reconvertirent en latifundistes (Brito, 1966). Ensuite, les ventes (enajenaciones) des terrains de la nation (baldios) prennent une certaine importance, et les données exhaustives sont disponibles (Le tableau 1) montre qu'elles représentent une attribution annuelle de près de $0,3 \%$ de la Superficie des exploitations agricoles (SEA) au long du $\mathrm{XIX}^{\mathrm{e}}$ siècle. À partir de 1900 apparaissent les adjudications, dons de terre par le Gouvernement, en principe au profit de petits producteurs, mais attribués majoritairement aux grands producteurs

Les dotations de la réforme agraire, à partir de 1960, sont examinées plus loin.

- Une voie de fait (occupation, déplacement de clôtures, expulsion violente des premiers occupants, etc.), informelle, souvent régularisée a posteriori par des figures telles que : La composición coloniale, qui légitimait, contre paiement d'une amende, la propriété d'un occupant qui avait la possibilité de réaliser la coûteuse démarche.
Sous la République, la vente des baldíos concernait souvent des terrains déjà occupés par l'acheteur.

$\mathrm{La}$ « régularisation de la tenure » des terrains de la réforme agraire, décrétée en 1979, est la dernière forme de cette légalisation de la possession informelle, elle sera examinée plus loin.

Ce processus a eu deux conséquences importantes pour la configuration actuelle des droits de propriété :

(i) La concentration de leur contrôle au profit d'un groupe restreint. Il a été longtemps (jusqu'en 1958), mis en œuvre par les petits groupes qui ont contrôlé le pouvoir (conquistadores coloniaux et caudillos républicains). Le nombre de titres délivrés par an est très réduit (tableau 1). La tenure est restée effectivement très concentrée : en 1961, au début de la période de réforme agraire au Venezuela, 1,3\% des exploitations contrôlaient $71,7 \%$ de la SEA.

(ii) Leur caractère informel. La voie illégale semble avoir été toujours importante. Mais il est difficile de la quantifier, les données sont peu précises. Le seul cas exhaustivement documenté est le programme de régularisation de la tenure des terres de la réforme agraire qui a couvert une superficie trois fois plus importante que la dotation directe pendant la même période (Delahaye, op.cit.). Ce programme mis en œuvre à partir de 1979, régularisait les droits de propriété obtenus sur celles-ci en dehors du cadre légal. Cette informalité de la formation des droits de propriété contribue à expliquer la fréquente absence de titre foncier. L'importance de la propriété informelle n'est d'ailleurs pas exclusive du Venezuela, elle a permis l'accumulation " entre les mains de ceux qui contrôlent la plus grande partie de la terre " en Amérique latine (Barraclough et Collarte, 1971). Deininger (op.cit.) relève dans le continent «une perception de faiblesse des droits de propriété » en remarquant que les normes informelles de tenure sont les 
Tableau 1. Evolution quantitative de la délivrance de titres fonciers depuis l'indépendance, selon les formes et les périodes indiquées

\begin{tabular}{l|l|r|r|r|r|r}
\hline \multirow{2}{*}{ Période } & \multirow{2}{*}{$\begin{array}{c}\text { Type de titre } \\
\text { foncier }\end{array}$} & $\begin{array}{c}\text { Nombre } \\
\text { de titres }\end{array}$ & $\%$ SEA* & \multirow{2}{*}{$\begin{array}{c}\text { ha } \\
\text { partal ha }\end{array}$} & $\begin{array}{c}\% \text { SEA } \\
\text { dans la } \\
\text { période }\end{array}$ \\
\cline { 3 - 5 } $1821-99$ & Ventes de baldíos & 8,4 & 0,28 (SEA1937) & 65040 & 7742 & 19,25 \\
\hline $1900-35$ & VAB & 30,4 & 0,21 (SEA1937) & 48271 & 1588 & 7,56 \\
\hline $1936-57$ & VAB & 6,0 & 0,03 (SEA1937) & 7496 & 1249 & 0,60 \\
\hline $1958-73$ & RA et TS & 11416 & 1,3 (SEA 1961) & 346782 & 30 & 20,80 \\
\hline $1974-82$ & RA et TS & 9260 & 1,5 (SEA 1981) & 460317 & 50 & 13,50 \\
\hline $1983-90$ & RA et TS & 7859 & 1,2 (SEA 1981) & 384162 & 48 & 9,60 \\
\hline $1993-98$ & RA et TS & 7228 & 1,0 (SEA 1997) & 303781 & 42 & 6,00 \\
\hline
\end{tabular}

Source : Delahaye 2001 et 2003 à partir de CENDES-CIDA, 1969, et des archives officielles (Cadastre rural, Institut agraire national, ministère de l'Agriculture).

Notes

RA et TS : réforme agraire et titres supplétoires. - VAB : ventes et adjudications de baldíos Les données de la réforme agraire pour 1991-1992 et 1998-2000 ne sont pas disponibles.

* SEA N = Superficie des exploitations agricoles pour l'année N (année du recensement agricole le plus proche pendant la période indiquée). Le recensement de 1937 couvre 26000000 ha, celui de 1997, 30000000 ha.

plus courantes. De Janvry et al. (2001) insistent sur le peu de résultats des actions gouvernementales pour mettre en place des régulations foncières formelles. Au Venezuela, une importante proportion des terres agricoles reste publique, souvent possédée informellement. Même si les données existantes ne permettent pas de la déterminer avec précision, on peut estimer qu'elle représente entre 50 et $70 \%$ de la SEA (Delahaye, 2001).

\section{Le cadre historique et régional}

Ce travail distinguera trois périodes essentielles depuis la conquête (Carvallo et Ríos, 1990).

- La conquête elle-même, qui fut longue et meurtrière au Venezuela où il n'existait pas d'État centralisé, sinon une multitude de communautés qu'il fallait soumettre l'une après l'autre (Watchell, 1970). Elle fut suivie par une période d'installation précaire et de formation des premières exploitations agricoles, une fois acquise la certitude qu'il y avait peu de métaux précieux exploitables.

- La période d'agro exportation, de la fin du $\mathrm{XVI}^{\mathrm{e}}$ siècle aux premières décennies $\mathrm{du} \mathrm{XX}^{\mathrm{e}}$ siècle. Carvallo et Ríos (op.cit.) caractérisent les types des exploitations qui se sont développées. Ces auteurs distinguent :

(i) L'hacienda de la zone côtière, plantation qui produit essentiellement pour l'exportation (tabac, indigo, cacao, puis café) et exige de grandes superficies du fait du caractère extensif des cultures et de la nécessité de fournir des lopins aux travailleurs, qu'ils soient esclaves, puis peones (travailleurs qui vivent dans l'exploitation et travaillent les terres du patron pour un salaire réduit ou pratiquement nul, pour compenser l'attribution du lopin). L'exploitant a un caractère de caudillo (chef militaire) dont les peones sont les soldats. Il participe aux constantes guerres civiles du XIX ${ }^{\mathrm{e}}$ siècle, pour le contrôle du pouvoir politique.

(ii) Le hato d'élevage des plaines (llanos), très extensif, qui, de ce fait, réclame lui aussi de grandes superficies (Carvallo, 1985). Son propriétaire est lui aussi un caudillo, dont les peones à cheval forment la cavalerie des guerres civiles.

(iii) Les caudillos des haciendas et hatos sont logiquement les principaux bénéficiaires des attributions de terrains par l'État. Le tableau 1 montre que la moyenne de superficie de ceux-ci est de près de 8000 ha. Il s'agit bien de formation de grandes 
propriétés, d'autant plus que des études locales (Delahaye, 1993) montrent que, dans de nombreux cas, cette dotation initiale a été considérablement agrandie par des opérations illégales réalisées tout au long des siècles suivants : c'est le jeu de l'informel évoqué plus haut.

(iv) Les exploitations de production familiale des massifs montagneux proviennent essentiellement de la dissolution des resguardos des communautés précolombiennes qui avaient subsisté. Elle fut mise en œuvre par la loi républicaine de 1821 (Arcila, 1973). Ces exploitations produisent pour l'autoconsommation et le marché, qu'il s'agisse d'exportation (de café, dès le XVIII ${ }^{e}$ siècle) ou du marché intérieur lorsque celui-ci se développe avec l'exploitation pétrolière et l'urbanisation à partir des années 1930.

\section{- La transition (1936-1957)}

Dans les années 1930-1940 se sont formées de nouvelles exploitations familiales commerciales, de taille moyenne, sur des terres publiques qui avaient peu été touchées par le développement agricole historique (Llambi, 1988). Elles sont dirigées par des producteurs d'origine urbaine ou bien immigrants (Espagnols, Italiens ou Portugais). Elles pratiquent une agriculture mécanisée et technologique sans rapport avec l'agriculture historique (l'exportation pétrolière minimise l'importance du café et du cacao : moins de $5 \%$ du total des exportations depuis les années 1930). Elles produisent pour le marché intérieur en pleine expansion (la proportion de population urbaine passe de $15 \%$ en 1920 à $67 \%$ en 1961).

Dans le même temps, la hacienda disparaît, affectée par la crise des plantations due à la faiblesse du marché mondial du café dans les années 30, puis par la réforme agraire à partir de 1958 (Hernández, 1988) ; elle va être remplacée par des petites et moyennes exploitations produites par la division des domaines par héritage ou sur le marché, et par des asentamientos ${ }^{3}$ de la réforme agraire ; le hato se modernise lentement, et sa superficie diminue (Carvallo, op.cit.) ; les exploitations familiales des massifs montagneux évoluent dans un cadre commercial, pratiquant de nouvelles cultures de maraîchage et fleurs (Llambi, op.cit.).

\section{- Les dernières décennies du XX siècle}

Elles suivent le début de la période dite démocratique, après l'expulsion du dictateur Pérez Jiménez (1958). Elles correspondent à l'expression vénézuélienne de la «vague de démocratisation » en Amérique latine, décrite par Collier et Mahonney (1995; 1997), qui empruntent le concept à Huntington (1991) ${ }^{4}$. Chonchol (1994) évoque pour ces mêmes années le « quatrième moment significatif dans la formation des systèmes agraires ».

- Une réforme agraire massive qui s'essouffle dans les dernières décennies

La Loi de réforme agraire de 1960 (LRA) marque un moment essentiel. Elle prévoit la dotation de terres (dans l'application, il s'agira pour les trois-quarts de terrains publics) (Delahaye, 1993) gratuite (pour les paysans sans terres ou les peones) ou payante (pour les moyens agriculteurs qui sont essentiellement les nouveaux producteurs au marché interne). Ces terrains ne peuvent être cédés sur le marché formel, mais vont faire l'objet d'un échange informel important (Delahaye 2001). La LRA a fait l'objet d'un impressionnant consensus. Votée à l'unanimité par le Congrès, elle présentait des aspects favorables pour les principaux groupes sociaux économiques :

Les hacendados (planteurs agro exporta-

3. Exploitations (parcellées ou collectives) sur les exploitations de la réforme agraire.

4. Pour cet auteur, une vague de démocratisation correspond à « un groupe de transitions de régimes non démocratiques à des régimes démocratiques qui se produisent pendant une période donnée et qui sont significativement plus nombreuses que les transitions dans l'autre sens pendant la même période. » 
Tableau 2. Moyennes annuelles des dotations de réforme agraire et totaux correspondants (1958-1998)

\begin{tabular}{l|c|c|c}
\hline \multicolumn{1}{c|}{ Années } & Nombre de familles dotées & Superficie dotée (ha) & \% SEA \\
\hline $1958-73$ & 11.312 & 331.840 & $1,25 \%$ SEA 1971 \\
\hline $1974-78$ & 1.097 & 173.596 & $\mathbf{0 , 6 6} \%$ SEA 1971 \\
\hline $1979-82$ & 19.228 & 785.085 & $\mathbf{2 , 5 0} \%$ SEA 1984 \\
\hline $1983-90$ & 7.692 & 340.828 & $1,17 \%$ SEA 1984 \\
\hline $1993-98$ & 6.254 & 303.782 & $0,87 \%$ SEA 1997 \\
\hline Total 1958-98 & 371814 & 13810945 & $46,3 \%$ SEA 1997 \\
\hline
\end{tabular}

Source : Delahaye (2001 et 2003) à partir des rapports annuels de I'Institut agraire national et du ministère de l'Agriculture, et de CENDES-CIDA 1969 (tome 2).

Note. Les données des années 1991-92 et postérieures à 1998 ne sont pas disponibles.

teurs) y trouvaient leur bénéfice dans les indemnisations qui les débarrassaient d'exploitations en pleine crise (Hernández, op.cit.). De leur côté, les hateros (éleveurs) en lente modernisation (Carvallo, 1985) ont pu résister à une réforme agraire qui a affecté le plus faible pourcentage de la SEA dans les régions de hatos où les demandes paysannes de terre présentées à l'Institut agraire étaient inférieures à celles des autres régions, en particulier du fait du nombre réduit de peones et de paysans sans terres (Delahaye, 2003). La dotation pour les nouveaux agriculteurs qui produisaient pour le marché intérieur était prévue dans la LRA, sous forme onéreuse, avec des délais de payement très généreux (vingt ans, sans intérêt). Les peones et paysans sans terre des régions de haciendas satisfaisaient leur principale revendication (exprimée historiquement par des invasions de terrain qui ont ponctué tous les changements de régime au $\mathrm{Xx}^{\mathrm{e}}$ siècle) (Delahaye, 2001). Enfin les patrons de l'industrie et du commerce voyaient dans la réforme agraire une possibilité d'élargir le marché intérieur aux paysans qui vont recevoir un crédit et vendre leur récolte.

Le rythme historique très bas de délivrance de titres fonciers est effectivement rompu : il passe de quelques unités à plusieurs milliers par an (tableau 1). La superficie concernée annuellement passe de moins de $0,3 \%$ à plus de $1 \%$ de la SEA. La réforme agraire est d'ailleurs impulsée à partir de 1959 par l'Alliance pour le Progrès constituée par les États-Unis pour contrer la possibilité de guérillas paysannes procastristes en Amérique Latine (Barraclough et Collarte, op.cit.). Elle est massive : entre 1958 et 2000, elle attribue 13800000 hectares $(45,6 \%$ de la SEA de 1997), suivant un rythme annuel relativement élevé (tableau 2), qui décroît sensiblement à partir des années 1970.

Le pic de 1979-1982 correspond à l'application du programme de « régularisation de la tenure » (décret de 1979), qui régularise les droits des producteurs occupant informellement des terres de l'Institut agraire national (IAN), soit par occupation, soit par achat illégal. L'IAN délivre des titres de régularisation qui correspondent aux mêmes droits que ceux des dotations. Ils ont aussi les mêmes destinataires : gratuits pour les « petits producteurs », ils sont onéreux pour les « moyens ». Si la réforme agraire a pourvu 371814 familles d'un terrain, selon les chiffres officiels, les enquêtes successives de l'IAN n'ont jamais dénombré plus de 160000 producteurs sur ses terres (Delahaye, 2001). La différence est due en grande partie aux ventes informelles de droits sur les parcelles.

Plusieurs aspects de la réforme agraire méritent l'attention, dans la mesure où ils peuvent offrir des explications pour la situation actuelle :

- La diminution des demandes de terre présentées par des paysans à l'IAN (tableau 3) explique partiellement le fléchissement de 
Tableau 3. Demandes paysannes de terres présentées dans les dossiers de l'Institut agraire national (1958-1998, moyennes annuelles)

\begin{tabular}{c|c|c}
\hline Années & $\begin{array}{c}\text { Nombre de paysans demandeurs } \\
\text { dans les dossiers }\end{array}$ & $\begin{array}{c}\text { Nombre de dossiers } \\
\text { présentés }\end{array}$ \\
\hline $1958-73$ & 4654 & 74 \\
\hline $1974-78$ & 1867 & 31 \\
\hline $1979-82$ & 986 & 19 \\
\hline $1983-89$ & 910 & 16 \\
\hline
\end{tabular}

Source : Delahaye (2001) à partir des dossiers de la direction des terres de I'IAN

Note. Un dossier correspond à une sollicitude concernant un terrain donné.

l'activité de dotation de la réforme agraire.

- La réforme agraire a peu pris en compte les terres publiques, malgré leur importance. Le slogan était : «la réforme agraire doit se réaliser en terres privées; en terres publiques, il s'agit de colonisation ${ }^{5}$. De ce fait, tandis que les terres publiques furent occupées par les moyens producteurs commerciaux qui visaient le marché intérieur, les paysans en furent marginalisés. Les droits correspondants furent souvent légalisés sous la formule des titres supplétoires qui étaient attribués aux occupants, suivant un processus assez expéditif . Entre $^{6}$ 1959 et 2000, 7000 titres ont été attribués pour une superficie de 2100000 ha, c'està-dire 7,5 \% de la SEA de 1997, dont la moitié correspond à des exploitations moyennes (Delahaye, 2003). Les responsables des politiques agraires n'ont pas pris en compte cette formation de droits sur terres publiques, malgré les protestations du Syndicat paysan national (CENDESCIDA, 1969).

- L'évolution de la structure de la tenure (indiquée dans les recensements agricoles) ne démontre pas la création de petites exploitations :

(i) Les petites exploitations (de moins de 50 ha) n'augmentent pas substantiellement

5. Voir CENDES-CIDA (1969), tome 1.

6. Ces titres reconnaissent la propriété de l'exploitant sur les améliorations, cultures, constructions, etc., qu'il a réalisées sur le terrain occupé. Les améliorations correspondantes peuvent être vendues légalement, l'État restant propriétaire du terrain. leur part du foncier ( $8 \%$ de la SEA en 1961; $10,7 \%$ en 1997). La réforme agraire semble avoir eu un résultat pratiquement neutre sur ce point : les dotations ont été compensées par les abandons ou cessions informelles de parcelles (Delahaye, 2001).

(ii) Les grandes (de plus de 1000 ha) voient diminuer leur importance relative (de 71,7\% de la SEA à 46,4 \%); cela est le résultat de l'action combinée des acquisitions de grands domaines par l'IAN et de la fragmentation des exploitations par le marché (Delahaye, 1996).

(iii) Les moyennes (de 50 à 1000 ha) passent à contrôler $42,9 \%$ de la SEA (20,3\% en 1961). Cette augmentation indique le dynamisme des nouvelles exploitations commerciales de taille moyenne qui produisent pour le marché intérieur.

Ce sont donc les moyennes exploitations qui se développèrent, bien qu'elles n'aient pas constitué l'objectif explicite de la réforme agraire. Elles ont plusieurs origines : fragmentation sur le marché des haciendas en crise (Hernández, 1988), achat informel de parcelles de la réforme agraire (Delahaye, 2001) et occupation des terres publiques par les nouveaux producteurs (Llambi, op.cit.).

- L'accès à la terre se réalise aussi à travers le marché foncier. Celui-ci existait régionalement depuis les premières années coloniales. Mais il s'est formé au niveau national au début des années 1960, lorsque, sur l'ensemble du pays (Delahaye, 2001) s'observe la condition définie par Braudel (1979) : 
«... fluctuation et unisson des prix entre les marchés d'une zone donnée ». La mise en œuvre de la réforme agraire a joué un rôle important dans sa formation (Delahaye 1993 ; 2001). Le marché légal concerne des terres privées ou des droits établis en terres publiques $^{7}$ (souvent formalisés par des titres supplétoires), et a redistribué une superficie largement supérieure à celle attribuée par la réforme agraire (entre 1958 et 1997, le pourcentage annuel de la SEA échangé sur le marché varie entre 5,1 et $14 \%{ }^{8}$, Delahaye, 2005). La superficie moyenne des terrains échangés sur ce marché légal diminue de 5 à 40 fois au long du $\mathrm{XX}^{\mathrm{e}}$ siècle, selon les régions, et le pourcentage de terrains divisés au cours d'une vente est supérieur à $30 \%$ (Delahaye, 2001), ce qui indique un rôle important du marché dans la restructuration agraire.

- Un marché informel, illégal, concerne les droits sur les terrains de la réforme agraire (RA), qui sont échangés sous la forme de bienhechurías (améliorations apportées aux terrains : cultures, clôtures, etc.) Ces cessions ont été soumises à réglementation dans la loi de réforme agraire de 1960, qui les rendait quasiment impossibles dans la pratique. Un décret de 1976 les a interdites sur la plus grande partie des terres de la RA. Ces interdictions n'ont pas été respectées; les cessions se sont réalisées informellement. Il existe peu de données à leur sujet, du fait de leur caractère illégal. Des enquêtes de terrain menées dans les asentamientos de la RA de la région d'Aragua ont obtenu des pourcentages de terres échangées annuellement

7. Non transférées à l'IAN (une importante fraction des terres publiques lui a été transférée depuis la fin des années 1960. Lorsqu'elles ont été transférées, les droits de leurs occupants ne peuvent être légalement vendus, ni faire l'objet de titres supplétoires, tout au moins jusqu'aux années récentes).

8. Ce pourcentage élevé inclut les ventes intrafamiliales simulées pour éviter les paiements de droits de succession (Delahaye, 1996) et les ventes sur les fronts de défrichement, où le marché est particulièrement actif (Delahaye, 2001). de 1 à $2 \%$, quelques années après leur fondation (Delahaye et al., 2003). Comparés avec les paysans bénéficiaires de la dotation initiale (ou leurs héritiers), les acheteurs sont dans une plus grande proportion domiciliés en ville, ont une profession non agricole, et sont souvent étrangers (immigrants espagnols ou portugais). Ce sont les caractéristiques que Llambi (1988) relève chez les « nouveaux agriculteurs » qu'il a étudiés.

La formation informelle des droits de propriété a donc joué un rôle essentiel, depuis la conquête jusqu'à la fin du $\mathrm{XX}^{\mathrm{c}}$ siècle. L'État l'a permise en ne contrôlant pas son patrimoine foncier, au bénéfice des groupes sociaux les plus proches de l'Administration à chaque moment historique. Cette formation a été en grande partie le produit de «pactes locaux » (Lemel, 1985) qui réunissent les dirigeants des « nouveaux producteurs » et les responsables locaux de l'application des lois foncières 9 .

\section{Les politiques foncières actuelles Continuité de la réforme}

La loi de terres et développement agricole (LTDA) de 2001 fut promulguée au milieu d'une période d'intenses débats agraires (Delahaye, 2002), lesquels se sont prolongés jusqu' aujourd'hui, avec plus ou moins d'intensité. Examinons tout d'abord le contexte, puis la mise en œuvre de la loi.

\section{Le rebond de la question agraire à partir de 1998}

La tendance déclinante aussi bien des demandes paysannes de terre que de l'importance de la question agraire dans les débats nationaux, apparaît avoir été brusquement renversée par les nombreuses occupations de terrains qui eurent lieu depuis la campagne présidentielle de Hugo Chávez Frías 1998, et par les mesures prises par le Gouvernement sur le plan agraire. Mais cette réflexion doit être nuancée.

9. Voir plusieurs exemples dans Delahaye (2001). 
Pendant et depuis la campagne électorale présidentielle de 1998, les occupations de terrains ont effectivement été fréquentes. Cela avait été le cas pour chaque changement de régime ou chaque élection présidentielle depuis 1936 (Troconis 1962 ; Delahaye 2001). Un examen des situations locales montre que, depuis 1998 , les occupations ont affecté essentiellement?

- Des terrains périurbains (environ $70 \%$ du total des occupations). Il s'agit de luttes menées par des urbains, pour obtenir un terrain afin d'y construire une maison Elles ne sont pas la conséquence de l'histoire agraire du pays, sinon de la déficience des gouvernements successifs pour résoudre la question du logement des secteurs les moins favorisés.

- Des terres publiques (états de Barinas, Portuguesa et Zulia) pour environ $20 \%$ du total des occupations ${ }^{10}$. Le manque d'intérêt des responsables de la réforme agraire antérieure pour cette formation de droits en terres publiques avait permis un contrôle croissant de ces dernières par les «nouveaux agriculteurs ». Depuis 1998, les nombreuses occupations paysannes d'exploitations situées en baldíos amènent à prendre en compte les terrains publics. Elles expriment une demande qui n'avait pas été écoutée dans les décennies précédentes. L'importance des transactions informelles en terres de l'Institut national des terres (INTi), créé par la LTDA, qui remplace l'IAN et a reçu son patrimoine foncier, confirme la nécessité de régulariser les situations de fait créées en terres publiques. La recrudescence des invasions de ces dernières manifeste l'intérêt des organisations paysannes locales à être partie prenante de cette régularisation à venir.

10. Les données sur les occupations ont été recueillies à partir de compilations des données parues dans la presse. Les statistiques officielles sur les redistributions foncières ne sont plus disponibles depuis 1998.
- La demande des terres de grands domaines privés (hatos et haciendas) s'est considérablement réduite, du fait de la diminution de leur importance relative sur la scène agraire, sauf dans quelques cas ponctuels. Les occupations de terres privées, qui l'expriment au niveau local, représentent un faible pourcentage $(10 \%)$ du total observé dans le pays.

Les invasions n'expriment donc pas une inversion de la tendance de la diminution de la demande paysanne de terre, observée depuis 1958 au niveau national. Il s'agit plutôt de l'expression d'une nouvelle réclamation, qui concerne des terrains périurbains, et des terres publiques : les expectatives créées par le changement de régime ont dynamisé l'expression de nouvelles formes de demandes de terre.

\section{La nouvelle configuration agraire}

Les mesures néolibérales appliquées par le Gouvernement vénézuélien à partir de 1989 ont rompu la quasi unanimité observée lors de l'approbation de la LRA de 1960 : les industriels non globalisés habitués à la protection gouvernementale, la majorité des agriculteurs (les subventions á l'agriculture furent supprimées à partir de 1989), une bonne partie de la classe moyenne laminée par l'inflation, se sentait menacée par les conséquences de la globalisation. Ce mécontentement permit l'élection du président Chávez.

À la campagne, le marché informel avait montré la possibilité de vendre les droits acquis sur la terre dans le cadre de la réforme agraire. De nombreux producteurs, ainsi que des habitants des villes, virent une possibilité d'obtenir par l'invasion un droit qui serait revendu dès que possible. Il n'existe pas de données fiables sur ce sujet, mais les articles de presse fournissent des exemples ${ }^{11}$.

11. Voir à ce sujet El Universal des 11 et 22 septembre 2008. 


\section{La mise en œuvre de la LTDA}

Les mesures agraires du Gouvernement élu en 1998 ont pour pivot la LTDA de 2001 (reformulée partiellement en 2005). Ses objectifs sont ambitieux (développement durable et planifié, dotation de terres, fin du "latifundio", incitation à la production dans les moyennes et grandes exploitations, perception d'un impôt foncier, etc.). Cependant ces objectifs ne peuvent être atteints, car les aspects concrets de son application n'ont pas été précisés. Cela crée une indécision dans la mise en œuvre des mesures. Voyons quelques exemples.

- Les exploitations doivent être certifiées comme « incultes », « améliorables » ou «productives ». Des terres incultes peuvent faire l'objet d'expropriation. Mais la loi n'établit pas les critères correspondants, ce qui laisse une grande marge d'appréciation aux fonctionnaires régionaux. Cette certification joue un rôle comparable à celui de la « fonction sociale » inscrite dans la LRA de 1960, mais celle-ci précisait les critères que devaient remplir les exploitations pour ne pas être expropriables.

- Le latifundio : c'est une forme de tenure qui a disparu avec le péonage dans les années 1960 ; l'attaquer maintenant (comme le fait la LTDA) n'a pas de sens. D'autant plus que cette loi le définissait à partir de la superficie, quand les caractéristiques du latifundio étaient les rapports de production basés sur la dépendance personnelle des travailleurs vis-à-vis du latifundiste (peonaje au Venezuela, huasipongo en Équateur, inquilinaje au Chili, etc.). Une révision de la LTDA a modifié en 2005 ce concept de latifundio et le définit à partir de critères techniques et économiques. Elle maintient cependant le terme, qui ne correspond pas à la réalité agraire actuelle.

- La loi prévoit la perception d'un impôt foncier par le Service national des douanes et impôts (SENIAT). Il s'agit de l'un des objectifs les plus importants de la LTDA, qui n'avait pas été inscrit dans la loi de 1960.
Mais il manque les éléments matériels indispensables pour sa perception: un cadastre mis à jour, une classification des sols, un registre fiable des propriétés. La direction du SENIAT reconnait de plus en plus que sa perception va être particulièrement problématique (voir en particulier la déclaration du directeur dans El Nacional, 28 mars 2005).

- L'interdiction de la vente, sans autorisation de l'INTi, des terres de la réforme agraire par leur bénéficiaire, inscrite la loi de 1960, se maintient dans la LTDA. Cette interdiction a fait l'objet de critiques acerbes des partis de l'opposition, qui l'accusent de ne pas respecter la « propriété » quand les mêmes partis la défendaient à outrance lors qu'ils étaient au pouvoir (Delahaye, 2002). Elle n'est pas plus respectée actuellement que sous le régime de la loi antérieure. $\mathrm{La}$ vente illégale de droits nourrit leur marché informel évoqué plus haut. Là encore, pour faire appliquer la loi, il est nécessaire de promulguer des textes qui permettent sa mise en œuvre dans la pratique. Des sondages au niveau local (García 2008) indiquent d'ailleurs que l'INTi délivre des autorisations de ventes d'améliorations sur ses terres, sans qu'il soit possible de les quantifier nationalement. C'est un retour au régime des premières années d'application de la loi de 1960.

- Les mesures d'attribution de terres : le manque de définitions nécessaires pour l'application de la LTDA, qui résulte de l'absence de règlements d'application, a mené à une paralysie progressive des actions agraires du Gouvernement, bien que l'article 5 de la loi (17 dans la reformulation de 2005) garantisse l'adjudication de terres au « secteur paysan », sans précision de forme juridique. Le Gouvernement a tenté dans les toutes dernières années de dépasser ce blocage en appliquant deux types de mesures :

(i) Les « chartes agraires ", non prévues par la LTDA, furent crées administrativement par la résolution 177 de l'INTi du 5 
février 2003. Elles octroient, à des groupes de paysans organisés, un droit d'occupation d'exploitations fondées par d'autres personnes sur des terres publiques, tandis que l'INTi résout la question des droits de propriété. Mais la superposition d'un droit nouveau sur un droit préexistant qui n'a pas été liquidé est une source probable de conflits à moyen et long terme. D'autre part, la charte a été parfois établie « au groupe organisé pour demander le terrain $\mathrm{X} »$, sans indication des noms des membres du groupe. Cela rend précaire le droit de propriété correspondant. Une formule semblable avait été déjà appliquée dans les années 1960 , ce qui créa des problèmes difficiles à résoudre sur le long terme (Delahaye, 2001). Le nombre de chartes agraires n'est pas connu avec exactitude. D'après des indications de presse, le rythme annuel d'attribution de cartes agraires est de l'ordre de 10.000 sur l'ensemble de la période 1998-2007. Les chiffres exacts ne sont pas accessibles. D'autre part une polémique s'est développée en 2005 sur leur validité juridique, et des déclarations de responsables agraires nationaux ont laissé entendre qu'elles ne seraient plus délivrées. Cependant des données isolées indiquent qu'elles continuent à être octroyées, tout au moins dans certaines régions (García, 2008). La superficie correspondante ne peut être évaluée, mais il semble s'agir en général de quelques hectares par charte. La révision des archives de quelques directions régionales de l'INTi (García, op.cit.) montre qu'une proportion importante des chartes, impossible à évaluer au niveau national, n'est pas retirée par leurs bénéficiaires, et n'est donc pas opérationnelle. D'autres sont comptabilisées plusieurs fois. Même si les estimations restent approximatives, il semble bien qu'il n'y ait pas de rupture avec le rythme annuel de dotation de la réforme agraire précédente.

(ii) La médiation agraire pour acquérir des terrains privés à l'amiable, a été appliquée beaucoup plus récemment, depuis septembre 2005. Elle correspond aux revendications paysannes sur des domaines privées, qui s'expriment ponctuellement dans certaines régions. Elle a eu jusqu'ici une portée très limitée : elle a permis des accords entre propriétaires fonciers et Gouvernement dans 16 exploitations qui correspondent à une surface de 169000 ha (selon des données de 2006 du service juridique de l'INTi), en principe en terres de propriété privée (en fait, les grandes exploitations ont souvent été fondées à partir d'un titre original sur 5 à 10000 ha, et avaient occupé au cours des ans une superficie au moins équivalente de terres publiques et même parfois de communautés ou de personnes privées (Delahaye, 2001) : l'INTi reconnaît une « réserve » au propriétaire, qui cède une part de « ses » terres, attribuée aux paysans organisés en coopératives. Les terres affectées (et/ou les améliorations qui leur ont été apportées par l'exploitant) sont indemnisées au prix du marché. Il s'agit donc d'une attribution limitée de terres; là encore, le rythme de la réforme agraire reste peu innovant par rapport à celui des années antérieures.

Les chiffres disponibles ne permettent pas de présenter les données d'attribution de terres au niveau régional, ni de délimiter précisément les dotations sur terrains privés ou sur terres publiques.

- L'affectation des terres à la réforme agraire : les déclarations des présidents successifs de l'INTi permettent d'évaluer à 3,5 millions d'hectares la superficie des terres affectées, essentiellement publiques. Dans El Universal du 21 mars 2006 («Le Charcote est vendu au Gouvernement »), le président de l'INTi déclare : «Au total nous avons récupéré ... 3,4 millions d'hectares » (il n'est pas indiqué si cette récupération concerne des terrains privés, ou bien des bienhechurías établies sur terres publiques, ce qui semble le plus probable). Le même fonctionnaire déclare : «En suivant la méthode de médiation agraire, nous avons récupéré 169000 hectares dans tous le pays 
et nous sommes en train d'en négocier 300000 de plus ». Ces derniers chiffres concernent des terres en principe privées ; ils sont considérablement inférieurs à ceux des terres publiques récupérées, qui correspondent à quelques millions d'hectares. La tendance à privilégier l'affectation de terres publiques, observée pour les décennies antérieures, continue donc à constituer l'axe des politiques foncières, avec un ordre de grandeur comparable à celui des premières années de la réforme agraire précédente. La récupération des terres de l'INTi est une opération essentielle : la superficie du patrimoine foncier de l'Institut n'est pas connue exactement, mais elle est certainement supérieure à la moitié de la SEA (Delahaye, 2001).

Les fundos zamoranos, ou la reprise d'une modalité collective

La division des terrains des communautés précolombiennes évoquée plus haut a fait disparaître la totalité de ces dernières comme modalités de propriété territoriale collectives $^{12}$ : il n'en restait que quatre en 1946 (Arcila, op.cit). Mais les dernières décennies ont été marquées par des tentatives d'établissement de formes collectives modernes de propriété et de production.

La dernière en date correspond aux fundos zamoranos $^{13}$, dont la finalité est de fonder des exploitations coopératives, qui ne sont pas prévues explicitement par la LTDA. Seul son article 4 fait allusion, en termes généraux, à l'établissement d'un « système coopératif, collectif ou communautaire » (la version de 2005 ajoute une mention générale au fundo collectif). Les articles 62-70, qui établissent les modalités de l'adjudication des terres ne spécifient aucune

12. Il faut cependant citer les communautés qui ont fui la conquête vers les forêts de l'Orénoque, et ont conservé en partie leur mode de vie, ainsi que les titres collectifs délivrés à des groupes ethniques depuis 1960, sans qu'il existe de données précises à ce sujet.

13. De Zamora, leader paysan du XIX ${ }^{\mathrm{e}}$ siècle. modalité d'attribution collective. Cependant, la création de fundos zamoranos a été évoquée dès les premières années du régime, et s'est matérialisée par la création de 85 coopératives sur 400000 ha selon le ministre de l'Agriculture ${ }^{14}$. Peu d'éléments objectifs d'évaluation existent à leur sujet. Il ne s'agit pas d'une tentative entièrement nouvelle au Venezuela, et il vaut la peine de s'intéresser à l'enseignement des expériences précédentes.

Il s'agit tout d'abord des « communautés agraires », des « coopératives de production qui commencent à fonctionner comme des fermes d'État » (Fernández y Fernández 1948). Elles furent créées par la Corporation vénézuélienne de développement (CVF) par la « junte civique et militaire » qui arriva au pouvoir par un coup d'État en octobre 1945, et réunissait le Parti action démocratique et un groupe d'officiers (qui, au moyen d'un autre coup d'État en novembre 1948, se débarrassa de ses alliés civils, commençant la dictature de Pérez Jiménez qui dura jusqu'à janvier 1958). Leur objectif, énoncé dans une résolution de la CVF de 1946 (Venezuela, ministerio de Agricultura y Cría, Comisión de reforma agraria, 1959) était de créer des fermes d'État, « jusqu'à ce que la communauté agraire puisse s'administrer elle-même. » Quatorze communautés furent fondées en 1947-48. Le rapport annuel de la CVF pour 1948 présente un tableau d'échec dans toutes les communautés (ibid.) : pertes considérables, production quasi nulle. Elles furent liquidées en 1950 par le nouveau régime. La sous-commission économique de la réforme agraire de 1959 (ibid.) l'ajoute « à la liste d'essais qui ont échoué », en considérant que l'administration des communautés par l'Administration publique est responsable de cet échec.

Une autre tentative correspond aux « entreprises paysannes » qui furent créées à l'occasion d'un nouveau changement de régime, après la chute de la dictature de

14. Voir El Universal, 3 octobre 2007. 
Pérez Jiménez en 1958. Ce furent elles aussi des exploitations collectives. Il n'était pas prévu de phase de transition pendant laquelle l'État serait responsable de leur administration. Elles n'étaient pas non plus mentionnées explicitement par la LRA de 1960, qui prévoyait cependant les dotations collectives. Elles atteignirent une certaine envergure au cours des années 1960 : le courant qui avait lancé les communautés agraires en 1945 était revenu au Gouvernement. Au moment de leur apogée, en 1968, elles étaient au nombre de 210, et comptaient 5000 membres (Soto, 1973). Mais les élections de 1968 installèrent des porteurs d'autres priorités aux niveaux dirigeants de l'IAN, et leur importance diminua. Il n'avait pas été publié d'évaluation à leur sujet. Un travail interne de l'IAN montre que leur nombre avait rapidement diminué, même si le chiffre de leurs membres se maintenait : 74 restaient inscrites sur les registres de l'IAN en 1971, avec 4780 membres (Venezuela, IAN, Departamento de promoción, Sección organizaciones económicas, 1971). Les données de ce travail indiquent que seulement une trentaine de ces entreprises montraient une certaine viabilité économique, dans la mesure où elles présentaient un taux de récupération du crédit de plus de $80 \%$. Seules quelques unes de ces entreprises ont survécu jusqu'aux années actuelles.

Il n'existe pas d'évaluation des causes de ces échecs, ni de l'évolution actuelle des fundos zamoranos, malgré les moyens importants mis à leur disposition.

\section{L'application de la LTDA : une continuité paradoxale}

Sur les aspects essentiels de la réforme, l'application de la LTDA semble donc montrer une continuité avec celle de la loi de 1960, malgré les déclarations en termes de rupture des dirigeants nationaux lors de la déclaration de guerre au latifundio des premières années de l'actuel Gouvernement. En ce qui concerne la certification des exploitations aussi bien que l'affectation et l'attribution des terres de la réforme agraire, les rythmes sont similaires à celui des quatre décennies précédentes. L'interdiction de la revente des parcelles attribuées se maintient, ainsi que son contournement dans la pratique au niveau local. La seule nouveauté est l'introduction de l'impôt foncier, mais il ne semble pas applicable dans l'état actuel du Registre de la propriété et du Cadastre.

\section{Conclusion \\ La persistance des caractères hérités du passé}

La concentration de la tenure, son informalité, et l'existence d'une ample extension de terres publiques, conséquences du cadre historique de formation des droits de propriété au Venezuela, se sont maintenues jusqu'à maintenant. Les efforts des gouvernements successifs, depuis l'instauration de la démocratie dans le pays jusqu'aux années actuelles, n'ont pas modifié substantiellement cette situation. La persistante tolérance manifestée par les successives administrations agraires visà-vis des régulations foncières informelles (marché parallèle des parcelles de la réforme agraire, occupation des terres publiques, jeu des pactes locaux), explique en grande partie cette continuité. L'examen de la mise en œuvre des politiques foncières menées au cours des dernières années confirme cette dernière, malgré les déclarations officielles de rupture.

\section{Remerciements}

L'auteur tient à remercier Luis Llambi pour ses conseils, et les deux rapporteurs anonymes de la revue pour leurs observations. 


\section{RÉFÉRENCES BIBLIOGRAPHIQUES}

Arcila E. (1973). Economía colonial de Venezuela. Caracas, Universidad Central de Venezuela, 2 tomes.

Barraclough S., Collarte J.-C. (1971). El hombre y la tierra en América latina. Santiago de Chile, ICIRA.

Braudel F. (1976-1979). Civilisation matérielle et capitalismo. Paris, Arthaud-Flammarion, 3 tomes.

Brito F. (1966). Historia económica y social de Venezuela. Caracas. Universidad Central de Venezuela, 2 tomes.

Carvallo G. (1985). El hato venezolano. Caracas, Tropykos.

Carvallo G., Ríos J. (1990). Análisis histórico de la organización del espacio en Venezuela. Caracas, CDCH, Universidad Central de Venezuela.

CENDES-CIDA. (1969). Reforma agraria en Venezuela. Caracas, Universidad Central de Venezuela, 10 tomes.

Chonchol J. (1994). Sistemas agrarios en América Latina. De la etapa prehispánica a la modernización conservadora. México, Fondo de Cultura Económica.

Collier R., Mahonney J. (1997). Adding collective Actors to Collective Outcomes: Labor and Recent Democratization in South America and Southern Europe. Comparative Politics, $\mathrm{n}^{\circ} 3$ p. 285-303.

Collier R., Mahonney J. (1995). Labor and Democratization: Comparing the first ahd third waves in Europe and Latin America.University of Berkeley.

Deininger K. (2003). Land Policies for Growth and Poverty Reduction. OxfordWashington, Oxford University PressWorld Bank.

Delahaye O. (2006). Tenencia de la tierra y desarrollo rural sostenible: algunos puntos para la reflexión en el caso venezolano. Agroalimentaria, $\mathrm{n}^{\circ}$ 23, p. 11-20.

Delahaye O. (2005). La transmission des droits fonciers au Venezuela au XX ${ }^{\mathrm{e}}$ siècle. Journées de la SFER: « Les institutions du développement durable des agricultures $d u$ Sud ». Montpellier , 7-9 novembre. Delahaye O. (2003). La privatización de la tierra agrícola en Venezuela desde Cristóbal Colón: la titulación (14932001). Caracas, Fondo Editorial Tropikos.

Delahaye O. (2002). La discusión sobre la ley de tierras: Espejismos y realidades. SIC, $\mathrm{n}^{\circ}$ 647, p. 350-354.

Delahaye O. (2001). Políticas de tierras de Venezuela en el siglo XX. Caracas, Fondo Editorial Tropikos.

Delahaye O. (1996). Una aproximación a la caracterización de las formas de transmisión de la propiedad de la tierra agrícola en dos distritos de Venezuela en el periodo 1900-1992. Revista de la Facultad de Agronomía, vol. 22, no 3-4, p. 1-14.

Delahaye O. (1993). Politiques foncières, luttes agraires et marché foncier au Venezuela (1958-1990). Thèse de Doctorat d'État en Lettres et Sciences Humaines. Université de Paris I, 2 tomes.

Delahaye O., Vásquez K.; Rivera H.; Montoya S.; Rodríguez J., González C. (2003). El mercado informal en las tierras del Instituto Agrario Nacional: algunas indicaciones en el distrito Zamora (Estado Aragua). Revista de la Facultad de Agronomía, vol. 29, ${ }^{\circ}$ 2, p. 233-242.

De Long B. (2005). Chavez's Agrarian Land Reform: More like Lincoln than Lenin. Washington, Memorandum to the Press, Council on Hemispheric Affairs. Fernández y Fernández R. (1948). Reforma agraria en Venezuela. Caracas, Corporación Venezolana de Fomento.

García F. (2008). Caracterización de las distintas formas de adjudicación de tierras por parte del Instituto Nacional de Tierras (INTi) en el estado Aragua (20032006). Maracay, Mémoire de fin d'études, Facultad de Agronomía, Universidad Central de Venezuela. 
Hernández J. de. (1988). La hacienda venezolana. Caracas, Tropykos.

Huntington S. P. (1991). The Thirst Wave: Democratization in the Late Twentieth Century. Norman, University of Oklahoma Press.

Janvry (de) A., Sadoulet E., Wolford D. (2002). The Changing Role of the State in Latin American Land Reform. In de Janvry A., Sadoulet E., Gordillo, G. (éd.), "Land Reform and Public Policy". Oxford, Oxford University Press.

Janvry (de) A., Gordillo G., Plateau, J.-P., et Sadoulet, E. (2001). Access to Land, Rural Poverty, and Public Action. Oxford, Oxford University Press.

Lemel H.-W. (1985). Land Titling: Conceptual, Empirical and Policy Issues. Land Tenure Center, University of WisconsinMadison.

Llambi L. (1988). La moderna finca familiar. Caracas, Editorial Acta Científica de Venezuela.

Soro D.-M., Colin P. (2004) Droits et gestion intra-familiale de la terre chez les migrants sénoufo en zone forestière de Côte-d'Ivoire. Le cas de Kongodjan, (sous-préfecture d'Adiaké). IRD, Document de travail de l'Unité de Recherche $095, n^{\circ} 11$.

Soto O. D. (1973). La empresa y la reforma agraria en la agricultura venezolana. Mérida, Instituto Iberoamericano de Derecho Agrario y Reforma Agraria. Troconis L. (1962). La cuestión agraria en la historia nacional. Caracas, Biblioteca de autores y temas tachirenses.

Venezuela, Institut Agraire National. (1970). Resultados del programa de investigación de la tenencia. Caracas. 3 volumes.

Venezuela, Institut Agraire National. (1976). Inventario nacional de tierras y beneficiarios de la Reforma Agraria. Caracas. 3 volumes.

Venezuela, Institut Agraire Nacional, Departamento de Promoción, Sección Organizaciones Económicas. (1971). Empresas campesinas. Caracas.

Venezuela, Ministerio de Agricultura y Cría, Comisión de Reforma Agraria. (1959). Informe de las Subcomisiones. Caracas. Volume II, tome 2.

Watchell N. (1970). La vision des vaincus. Paris, Gallimard. 